Théologiques

Théologiques

\title{
Le Dieu des possibilités : l'immanence et la transcendance repensées
}

\section{Rosemary R. Ruether}

Volume 8, numéro 2, automne 2000

Le pouvoir

URI : https://id.erudit.org/iderudit/005027ar

DOI : https://doi.org/10.7202/005027ar

Aller au sommaire du numéro

\section{Éditeur(s)}

Faculté de théologie de l'Université de Montréal

\section{ISSN}

1188-7109 (imprimé)

1492-1413 (numérique)

Découvrir la revue

\section{Citer cet article}

Ruether, R. R. (2000). Le Dieu des possibilités : l'immanence et la transcendance repensées. Théologiques, 8(2), 35-48. https://doi.org/10.7202/005027ar

\section{Résumé de l'article}

Dans cet article, Rosemary Ruether répond au Psalm of Anger to aPatriarchal god en interrogeant le concept de Dieu sousjacent à ce cri. Sapropre vision de Dieu est celle d'une matrice divine sousjacente à toute laréalité et non celle d'un Dieu patriarcal qui justifie la domination desfemmes. Cette vision de Dieu comme matrice divine soulève cependant laquestion de la relation entre la transcendance et l'immanence divines.Ruether rejette la division entre l'immanence et la transcendance quiconsidère Dieu soit comme un pouvoir distant à l'extérieur et au-dessus dela création, soit comme la fondation immanente de ce qui existe etjustifierait du coup les systèmes de domination existants. Ce dont nousavons besoin, c'est d'une synthèse dynamique de l'immanence et de latranscendance qu comprenne Dieu à la fois comme " radicalement autre "que tous les systèmes d'injustice et comme plus près de nous que nous ne lesommes à nous-mêmes. Cette vision sous-tend aussi la théologieécoféministe qui considère tous les êtres comme vivant et liant une relationactive Je-Tu les uns avec les autres. Dieu est la matrice dynamique des interrelationsentre tous les êtres. 


\section{Le Dieu des possibilités : l'immanence et la transcendance repensées}

Rosemary R. RUETHER *

Le Psaume de la femme abusée est le puissant cri de rage d'une femme qui demande : "Où étais-tu, Dieu, lorsque cela m'arrivait? " C'est la clameur de celle à qui on a appris que Dieu doit essentiellement être perçu comme une figure d'autorité mâle qui est en contrôle de tout ce qui se passe et qui juge les femmes comme étant celles qui ont introduit le péché dans le monde en désobéissant à Dieu. Dans la théologie chrétienne classique, les femmes ont été créées subordonnées à l'autorité masculine depuis le commencement, mais les femmes sont devenues rebelles, causant ainsi la chute de l'humanité dans le péché et la mort. Dieu a donc rétabli l'ordre social en mettant les femmes dans une position d'assujettissement coercitif par rapport à l'homme.

Les femmes seront sauvées en se soumettant à l'autorité masculine, même si celle-ci est injuste et violente. En souffrant ainsi plutôt que de résister à l'autorité de l'homme, les femmes seront sauvées. Cette théorie, développée par saint Augustin et reprise dans la tradition chrétienne classique par des théologiens dominants tels que Calvin au $16^{\mathrm{e}}$ siècle, justifiait en fait la violence contre les femmes. Lorsqu'une femme est battue, plutôt que de remettre en question l'autorité de l'homme, elle doit d'abord se demander ce qu'elle a fait

* Rosemary Radford Ruether est une théologienne éco-féministe catholique. Elle est professeure Georgia Harkness de théologie appliquée au Garrett-Evangelical Theological Seminary et membre du corps professoral des cycles supérieurs de Northwestern University à Evanston, Illinois, USA. Elle est auteure ou éditrice de trente-cinq livres et de nombreux articles portant sur les relations entre la théologie, le féminisme, l'écologie et la justice sociale. 
de mal et qui justifierait une telle violence. Ce Psaume est, implicitement, une remise en question de ce concept de Dieu.

Un tel concept de Dieu devrait, en effet, être repoussé et rejeté; et pourtant, je me sens moi-même un peu loin de cette protestation, car ce concept ne m'a jamais vraiment été présenté lorsque j'étais enfant. Je ne suis pas sûre de ce qu'était exactement ma vision de Dieu pendant mon enfance, mais je n'ai jamais eu l'idée que Dieu était quelqu'un qui justifierait la violence dirigée contre moi, ou même une limitation de mes possibilités en tant que femme. Peut-être est-ce grâce à ma mère, qui m'a toujours donné l'impression que j'étais une personne spéciale, aimée de Dieu et que je pouvais faire tout ce que je désirais afin de développer toutes mes possibilités.

Toutefois, il y avait un certain présupposé que Dieu était une sorte d' " intelligence » (mind) mâle désincarnée qui existait en dehors de la création dans un "espace " hors du cosmos. Lorsque j'avais à peu près 18 ans, j'ai commencé à remettre sérieusement en question l'existence d'un tel Dieu. Ce Dieu distant et en dehors de la réalité n'avait aucun sens pour moi. Je ne pouvais pas ressentir l'expérience d'un tel Dieu, et j'ai donc commencé à remettre en question son existence. Mon propre sens de Dieu ressemblait plutôt au «fondement de l'Être », bien que je ne connusse pas ce langage. Je crois qu'il s'agit là de la compréhension instinctive de Dieu vers laquelle tendent particulièrement les féministes. Pour moi, cela concordait avec mon expérience de ma mère et de ses amies comme des femmes judicieuses qui soutenaient mon plein potentiel en tant que petite fille. Cette compréhension de Dieu a récemment été attaquée par des théologiens chrétiens traditionnels comme étant «immanentiste » et donc " hérétique ".

Qu'est-ce qui est en jeu dans ce débat entre théologies féministes et traditionnelles concernant la relation entre l'immanence et la transcendance de Dieu? Les théologiennes féministes sont souvent perçues et se perçoivent elles-mêmes comme revendiquant une compréhension immanente de Dieu. Même si elles déclarent que leur compréhension de Dieu est à la fois transcendante et immanente, les personnes qui, dans l'Église, s'opposent à la théologie féministe affirment fréquemment que leur vision de Dieu est en fait uniquement immanente ou même "panthéiste ", et qu'il lui manque la transcendance essentielle à l'authentique vision biblique de Dieu. On présuppose ici que les 
compréhensions immanentes de Dieu sont fausses, "païennes ", et cela prouve que la théologie féministe est hérétique.

Cet ensemble de présuppositions a été exprimé lors d'une rencontre fortuite qui a eu lieu récemment entre une amie théologienne féministe et l'archevêque local lors d'une réception (je ne mentionnerai pas leurs noms). La femme lui a été présentée comme une théologienne féministe de premier plan, enseignant dans une université catholique de l'endroit. L'archevêque a reculé d'un pas et a déclaré : "La théologie féministe, c'est de l'immanentisme et l'immanentisme a été condamné comme hérésie. » Fin de la conversation! En supposant que l'archevêque eût été ouvert à la discussion, comment aurait-on pu analyser ses présupposés et commencer à ouvrir d'autres possibilités pour réfléchir à la relation entre l'immanence et la transcendance de Dieu?

L'autre facette de ce débat se déroule parfois entre les féministes chrétiennes et les éco-féministes et "théalogiennes " post-chrétiennes. Celles qui s'intéressent à l'éco-féminisme affirment souvent que toute idée de transcendance est fausse. Les visions transcendantales de Dieu sont intrinsèquement patriarcales, aliénantes et coercitives. Une vision transcendantale de Dieu signifie un Dieu hors du monde et qui le gouverne d'en haut, et non un Dieu présent dans le monde et par celui-ci. La transcendance est la projection sur Dieu des schémas sociaux d'oppression des femmes, des pauvres et de la terre.

Ces penseures féministes présupposent que si nous devons recouvrer une compréhension du divin qui puisse guérir nos relations interpersonnelles en tant qu'hommes et femmes, personnes du premier et du tiers-monde, Blancs et Noirs, et humains avec la terre, nous devons rejeter ce modèle transcendantal du divin. Nous devons soutenir une compréhension du divin comme étant la source et la puissance de vie dans et à travers toutes choses, qui ne soit pas séparée de nous-mêmes et de l'ensemble du monde naturel. Les théalogiennes, telle que Carol Christ, voient le divin essentiellement comme une personnification féminine. "Elle est en nous et en toutes choses ", ou même " elle est nous et toutes choses ${ }^{1}$. Toute séparation du divin d'avec la nature est considérée comme étant automatiquement un principe de domina-

1. Carol P. CHRIST, Laughter of Aphrodite: Reflections on a Journey to the Goddess, San Francisco, Harper and Row, 1987. 
tion et d'oppression. Manifestement, l'archevêque et la théalogienne se font face ici avec des anathèmes mutuels.

Il me semble que les féministes chrétiennes essaient de trouver leur chemin entre ces oppositions. Elles cherchent à maintenir ensemble la transcendance et l'immanence, et de manière à ne pas les séparer de façon dualiste. En effet, c'est cette séparation dualiste de l'immanence et de la transcendance qui est la racine du problème. Aussi longtemps que nous continuerons à présumer que la transcendance signifie rupture et distance et l'immanence une réduction à ce qui existe actuellement, aussi longtemps que nous associerons la première à la masculinité, à l'intelligence (mind) et à l'esprit et la seconde à la féminité, au corps et à la matière, nous ne pourrons pas nous sortir de cette impasse.

Dans mon propre cheminement théologique, je me sens depuis longtemps à l'aise avec une compréhension du divin comme étant la puissance et la source de vie au-dessous, autour et au travers de toutes choses. La célèbre déclaration de saint Paul, dans le livre des Actes, selon laquelle Dieu est celui en qui nous « avons la vie, le mouvement et l'être » (Actes 17,28) s'accorde avec mon expérience. La vision de Dieu comme un « vieil homme blanc et barbu » gouvernant la terre de l'extérieur ne m'a jamais semblé réelle.

Cette intuition s'est incarnée pour moi de manière dramatique lors d'une expérience que j'ai vécue lorsque j'avais environ dix-huit ans. Depuis quelque temps, je réfléchissais intensément à la signification de l'existence de Dieu. Un jour, j'ai vécu comme une sorte de "rêve éveillé " où je me voyais dans la vaste antichambre d'un château devant une porte fermée. J'ai ouvert la porte et j'ai constaté qu'elle donnait sur un escalier en colimaçon. Lorsque je suis arrivée en haut de l'escalier, j'ai trouvé une autre antichambre et une autre porte fermée. J'ai ouvert la porte et j'ai trouvé un autre escalier. Cela a duré pendant un certain temps, alors que j'ouvrais des portes successives et montais des escaliers successifs.

Finalement, je me suis retrouvée dans une antichambre face à ce que je sentais intuitivement être la dernière porte. Pour une raison ou pour une autre, je savais que derrière cette porte se trouvait la salle du trône de Dieu. Tremblante d'anticipation, j'ouvris toute grande cette dernière porte et j'entrai dans la salle du trône. À l'intérieur se trouvait un grand trône, mais je vis qu'il était vide! En réfléchissant à cette expérience, je 
me suis rendu compte que cela signifiait que Dieu ne se trouve pas au sommet d'une série de montées, toujours plus haut, vers le plus haut point de commandement au-dessus de tout le reste. La salle du trône au sommet de la tour du château est vide. Dieu n'est pas là.

Alors, où est Dieu? Mon intuition me disait : " Cherche Dieu, non en haut, mais en bas. Non dans les salles du trône du pouvoir et de la domination, mais à l'intérieur et au travers de ce qui nourrit la vie quotidienne. " Voilà la compréhension de Dieu qui rejoint mes sentiments, ma compréhension et mon être, qui rejoint mon être même tout entier. Voilà comment je fais l'expérience de Dieu lorsque je prie, lorsque je cherche à connaître sa présence. J'ai déjà parlé, dans mes écrits, de cette compréhension de Dieu en tant que " matrice divine ». Le langage employé par Tillich pour parler de Dieu comme le « fondement de l'être » me semble indiquer une compréhension similaire de Dieu.

La compréhension de Dieu en tant que matrice divine est-elle adéquate? Ou bien passe-t-elle à côté d'une grande vérité sur Dieu qu'expriment les images de Dieu comme régnant sur un trône à partir d'en haut? Et pourtant, je trouve impossible d'expérimenter cette vision de Dieu ou d'y croire vraiment. J'ai vu que la salle du trône est vide. Elle peut être remplie de rois, de chefs militaires, de directeurs généraux de sociétés internationales, mais elle est vide de Dieu. Dieu n'est pas là. L'Église a beau continuer à chanter que Dieu est Roi des Rois et Seigneur des Seigneurs, le trône reste inoccupé.

Cependant, je rejette la conséquence voulant que la compréhension de Dieu comme matrice divine soit simplement de l'immanentisme, au sens d'une réduction du divin à ce qui est. Car ce qui est, c'est précisément le monde gouverné par les chefs militaires et les directeurs généraux de sociétés internationales. Il y a quelque chose de faux dans l'identification de la transcendance avec le Dieu de la salle du trône. Dieu matrice divine n'est pas immanence contre transcendance, mais est à la fois immanent et transcendant, alors que le Dieu de la salle du trône démontre une fausse idée de la transcendance, une représentation de la relation de Dieu avec la création d'après le modèle de gouvernants oppressifs dominant les gouvernés.

Ce qu'on retrouve ici est une confusion fondamentale dans notre langage théologique quant au sens de l'immanence et de la transcendance, une confusion qui est souvent partagée par les féministes et les 
anti-féministes, par les théalogiennes aussi bien que par l'archevêque. Tant les théalogiennes que l'archevêque sont pris dans un faux dualisme où chacune des parties s'accroche à une demi-vérité déformée. L'archevêque pense que Dieu ne peut être vraiment Dieu que s' " Il » est compris comme étant spatialement en dehors de toutes choses créées et comme les gouvernant à partir d'en haut. Les théalogiennes présument que, pour que le divin soit principe de vie et source de tout, il doit être entièrement à l'intérieur du monde naturel, dans le sens où il est identique au monde et à la nature telles qu'ils sont actuellement.

Ce que les théologiennes féministes cherchent à affirmer, en insistant sur l'immanence divine, est une compréhension holistique de Dieu qui ne prenne pas pour modèle le dualisme de l'intelligence (mind) contre le corps, de l'esprit contre la matière, de l'homme contre la femme. Dieu est le pouvoir créateur qui est véritablement la source de toute vie dans sa plénitude et sa bonté. Cependant, les théologiennes féministes cherchent aussi un Dieu qui puisse nous libérer du patriarcat, nous libérer des systèmes oppressifs du monde qui nous entoure et qui ont constitué et constituent encore une si large part de l'histoire de l'humanité. La quête du Dieu libérateur, du Dieu des nouvelles possibilités est-elle en conflit avec la compréhension de Dieu comme source de tout ce qui est? L'idée de Dieu comme matrice divine, comme fondement de l'être, réduit-elle Dieu à ce qui existe actuellement, sacralisant par là les choses telles qu'elles sont?

Afin de sortir de cette impasse dualiste et de faire l'expérience d'un Dieu qui soit à la fois holistique et libérateur, nous devons nous libérer des dualismes esprit (mind)-corps, extérieur-intérieur, qui ont été encodés dans les concepts chrétiens de transcendance et d'immanence. Nous devons aussi remettre en question l'identification de la masculinité au côté transcendant et de la féminité au côté immanent de cette polarité. La transcendance de Dieu n'a rien à voir avec le fait d'être masculin, à l'extérieur, lointain et désincarné. L'immanence de Dieu n'a rien à voir avec le fait d'être féminine, à l'intérieur, proche et corporelle. Ces dualismes faussent la tension fondamentale de l'existence humaine entre ce qui est actuellement et ce qui devrait être, entre l'existence présente avec ses distorsions oppressives et les possibilités libératrices.

Je reprends ici une idée que j'ai apprise des écrits de Dorothee Soelle. La transcendance de Dieu signifie sa liberté radicale envers tous les systèmes humains de distorsions oppressives, de péché et de 
mensonges ${ }^{2}$. L'immanence de Dieu signifie sa présence libératrice en nous, au travers de nous et au-dessous de nous, présence qui nous donne le pouvoir de nous libérer de cette réalité oppressive de péché et de mensonges. Les deux, en fin de compte, ne font qu'un, c'est-àdire que Dieu est puissance et présence libératrices comme Quelqu'un qui est radicalement libre envers nos systèmes de péché et de mensonges et qui est plus près de nous que nous le sommes de nous-mêmes.

Le dieu de la salle du trône, vu comme le fondateur des systèmes d'oppression, n'est, par définition, ni transcendant ni libre envers ces systèmes, mais immobilisé en eux et défini par eux comme leur sommet et leur source mêmes. Un tel dieu est l'idole des systèmes d'oppression. Cela signifie que le dieu patriarcal, le dieu défini comme masculinité-esprit-extériorité, en excluant féminité-corps-présence, manque de transcendance authentique. Un tel dieu est plutôt la créature des idéologies et des systèmes de pouvoir humains, mis en place afin de justifier la domination des hommes sur les femmes, de la classe dirigeante sur les pauvres, des puissants sur les impuissants.

Tous ceux et celles qui cherchent une véritable libération doivent rejeter cette idole de la fausse transcendance, mais le fait d'adopter l'envers de ces mêmes dualismes et d'identifier le divin au féminin, au corps et à l'ici et maintenant ne nous aide nullement. Une telle immanence est dangereuse, pour les victimes de l'oppression et pour nous tous et toutes, car, par voie de conséquence, elle rend naturels les systèmes de domination. Elle sacralise sans discernement ce qui existe comme étant l'œuvre et la volonté de Dieu. Ce dont les personnes opprimées et nous tous et toutes avons plutôt besoin, c'est un Dieu de libération qui peut transformer ce qui est et nous libérer des systèmes de péché et de leurs idéologies qui se sacralisent elles-mêmes.

Il y a quelques années, Rita Gross, une théologienne féministe bouddhiste, a fait une distinction utile lors d'une discussion à l'American Academy of Religion portant sur l'immanence et la transcendance ${ }^{3}$. Gross a suggéré que nous devrions peut-être considérer la transcendance et l'immanence comme des expériences subjecti-

2. Dorothee SOELLE, "God Language and Patriarchy" in COELI International (Été 1992), p. 1-5.

3. Rita M. GROSS, «Immanence and Transcendence in Women's Religious Experience : A Non-Theistic Perspective ", dans Arvind Sharma et Katherine 
ves plutôt que comme des caractéristiques objectives ou ontologiques du divin. La transcendance, dans l'expérience religieuse, consiste dans les expériences d'exaltation qui nous permettent de nous élever audelà des schémas triviaux et oppressifs de l'existence présente et de toucher des possibilités transformatrices et libératrices. Ce sont précisément les personnes auxquelles la société dit de s'installer dans les aspects triviaux et oppressifs de la réalité présente, c'est-à-dire les femmes, qui ont le plus désespérément besoin de telles expériences de transcendance.

Que sont ces expériences de transcendance? Celles-ci peuvent nous arriver dans des expériences d'une beauté à couper le souffle, liées à la nature, à l'art et à la musique, qui nous élèvent au-delà de l'ordinaire. Celles-ci peuvent également être des expériences de dissonance cognitive bouleversantes qui nous forcent à rejeter le présupposé que les réalités oppressives sont "normales » et acceptables, en nous mettant en contact avec notre potentiel le plus profond de plénitude de vie. Nous pouvons trouver de telles expériences dans la prière profonde et attentive et dans des relations profondes et attentives avec les autres, autant avec les autres humains qu'avec les autres créatures de la terre. Les expériences de transcendance sont intrinsèquement heureuses, inattendues, imméritées, gracieuses. Elles nous viennent d' " au-delà " d'où nous " sommes ", et pourtant, elles nous mettent en contact avec ce que nous "sommes" plus véritablement. Elles sont la grâce divine et notre vraie « nature ».

Dans son article, Gross suggérait que c'est uniquement lorsque nous nous sommes engagés dans un long et profond cheminement d'expériences transcendantes et transformatrices, lorsque nous avons passé quelque temps à démêler ce qui est authentique et vivifiant de ce qui est illusoire et violent, que nous pouvons risquer des expériences d'immanence, c'est-à-dire expérimenter le divin comme la source sacrée de la vie quotidienne en nous et autour de nous. L' " immanence prématurée » est dangereuse, en particulier pour les femmes, car elle risque de sacraliser les schémas oppressifs mêmes dont nous avons besoin d'être libérés.

K. Young (éd.), The Annual Review of Women in World Religions, vol. 5, New York, SunY Press, 1999, p. 62-79. 
La compréhension de Dieu dans la théologie de libération écoféministe doit être radicalement transcendante, au sens de radicalement libre, la source même de la liberté envers tous les systèmes de péché et de mensonges. C'est uniquement lorsque nous sommes profondément en contact avec le dieu de la liberté libératrice, lorsque nous sommes profondément transformés grâce au dieu de liberté, que nous pouvons aussi embrasser Dieu comme Quelqu'un qui est plus près de nous que nous le sommes nous-mêmes, qui est au-dessous, autour et au travers de toute chose, la source de vie et du renouvellement de la vie. Le créateur de la vie est en même temps le Dieu des possibilités.

Cette compréhension de Dieu en tant que présence transformatrice, transcendance immanente et immanence transcendante, doit aussi transformer notre façon dualiste de séparer la nature de l'histoire, la création de la rédemption. Comme la tradition chrétienne l'a toujours affirmé, à l'encontre du dualisme marcionite, le Dieu qui crée le monde n'est pas autre que le Dieu qui rachète le monde. Cela signifie que Dieu comme créateur ne peut être identifié au fondateur des systèmes d'oppression. Les systèmes d'oppression sont plutôt des distorsions profondes de l'espérance de Dieu à l'égard de la création et à l'égard du propre potentiel et de la " nature véritable » de la création. La Rédemption est l'accomplissement de la Création, la manifestation achevée de son être véritable et de l'espérance de Dieu.

La division entre la nature et l'histoire, et l'identification des faux dieux «païens " aux "dieux de la nature » par opposition au "vrai Dieu biblique de l'histoire » ont été popularisées par la théologie allemande du $19^{\mathrm{e}}$ siècle et par la théologie néo-orthodoxe dans la première moitié du $20^{\mathrm{e}}$ siècle. Mais je crois que cette séparation fausse la perspective biblique. Bien que Dieu soit vu comme créant la nature plutôt que comme une expression de celle-ci, il y a néanmoins dans la Bible un vif sentiment de la présence de Dieu dans la nature.

La nature que Dieu crée est vivante et noue avec Dieu une relation pleine de vitalité. Dieu prend plaisir dans les animaux, les plantes, les collines et les ruisseaux que Dieu crée, et ces derniers répondent à ce plaisir par la louange et la réjouissance. La bénédiction divine inonde la terre comme une " eau douce ", les forêts du Liban bondissent comme un veau, les collines se ceignent de joie, les prés se revêtent de troupeaux, les vallées se parent de blé, ensemble elles et ils crient et 
chantent de joie (Psaumes 29,6; 65,9-13). Ce langage n'est pas simplement de la "poésie vide ", mais exprime l'expérience vitale d'une relation Je - Tu entre Dieu et la nature dans la spiritualité hébraïque.

Dans la pensée hébraïque, ce ne sont pas simplement les événements de l'histoire humaine, mais aussi les événements de la nature qui sont des expressions de la présence de Dieu dans la création. Dieu est présent et dans la bénédiction et dans le jugement. Autrement dit, tout ce qui se passe est un événement enraciné dans une relation à Dieu. La nature n'est pas "statique ", par opposition aux événements humains perçus comme changeants et ouverts à la nouveauté, mais c'est toute la création qui est historique. Le péché humain cause l'injustice dans les affaires humaines, mais pollue également la terre. La terre se languit dans la sécheresse et soupire après la bénédiction que constitue la pluie. Mais lorsque les humains retournent à l'obéissance à Dieu, il y a la justice et la paix dans les affaires humaines, mais aussi la paix entre les humains et la nature, et au sein de la nature ellemême. La nature et l'humanité s'épanouissent ensemble.

Cette vision serait problématique si elle était comprise de façon mécanique, blâmant les victimes pour les catastrophes naturelles. Mais on peut la lire comme une intuition profonde de la relation entre l'injustice et l'appauvrissement de la terre. Cette relation est devenue évidente dans la pensée écologique actuelle. Le colonialisme, par exemple, réduit les peuples indigènes en esclavage et s'empare aussi de leur terre. Il exploite leur travail ainsi que la terre pour le profit de la minorité colonisatrice. Le résultat est une terre dévastée et des communautés humaines dévastées : sol arable emporté, lacs et cours d'eau pollués, forêts pluviales détruites, ce qui affecte le climat planétaire, pauvreté et violence dans les vies humaines; une histoire racontée de façon dramatique, par exemple, dans le livre d'Eduardo Galeano, Open Veins of Latin America : Five Centuries of the Pillage of a Continent (1973).

Ces désastres manifestent l'injustice; mais ils ne sont pas euxmêmes justice divine. Car ce sont les victimes qui souffrent le plus des effets de ces désastres, tandis que les personnes qui exploitent d'autres humains ainsi que la terre continuent d'en tirer profit. Ces personnes aussi en paieront peut-être un jour le prix, mais uniquement lorsque tout le système s'écroulera comme un château de cartes. Alors, nous serons tous et toutes punis, mais cela n'est pas la rédemption. La 
rédemption doit joindre, à de nouvelles relations entre les humains, de nouvelles relations entre les humains et la terre. La justice doit être comprise comme éco-justice, la relation intime entre la façon dont nous nous traitons les uns les autres et la façon dont nous traitons la nature.

L'éco-justice signifie une transformation profonde de tout le système de relations entre nous et avec la terre, passant d'un système de violence et d'exploitation à un système qui peut nourrir, à tous les niveaux, des relations qui donnent la vie : entre les classes sociales, entre les races, entre les humains, et entre les humains et la terre. L'intuition éco-féministe est que les relations homme-femme sont à la fois des symboles et des expressions de ce tissu de liens existant entre les humains et entre les humains et la nature.

La façon dont nous pensons Dieu est cruciale à la transformation de notre pensée concernant tout ce tissu de relations. Dieu se situe dans une inter-relationalité dynamique avec toute sa création, et pas seulement avec les humains. Les humains constituent cependant un point central de cette interrelation, même si nous sommes arrivés tard sur cette planète et que notre avènement en tant qu'espèce dominante soit encore plus récent, c'est-à-dire au cours des derniers millénaires. Pourtant, à mesure que nous devenons de plus en plus capables de dominer et de contrôler la nature, nous en sommes venus à imaginer que nous lui étions transcendants, que nous étions indépendants d'elle.

Il s'agit là d'une conscience fausse, car quelle que soit l'ampleur de notre impact global, celui-ci se fonde encore totalement sur les processus terrestres vivifiants que sont la lumière du soleil, le sol et la photosynthèse réalisée par les plantes vertes. Nous avons le choix, soit d'utiliser nos connaissances et notre pouvoir pour nous harmoniser avec les processus qui permettent la viabilité de la terre, soit de nous détruire en tant qu'espèce et de détruire un bon nombre des créatures terrestres avec nous. Nous ne pouvons être rachetés que sur la terre et de la terre, non en dehors d'elle et contre elle. Il est crucial de repenser notre compréhension de la relation entre Dieu, les humains et la nature précisément parce que la pensée occidentale a modelé son concept de transcendance divine sur cette conscience fausse de notre autonomie envers la nature.

Certaines théologiennes éco-féministes, telle la Brésilienne Ivone Gebara, ont développé ce sens de l'inter-relationalité de toutes choses 
afin de reconstruire la compréhension de Dieu en tant que Trinité. Pour Gebara, le Dieu trinitaire est la matrice nourrissante de la relationalité. Le Dieu trinitaire ne signifie pas une relation séparée, enfermée sur elle-même de deux ego masculins désincarnés l'un avec l'autre, médiatisée par l'Esprit. La Trinité symbolise et exprime plutôt la dynamique de la vie elle-même en tant que processus de créativité inter-relationnelle vitale ${ }^{4}$.

La vie en tant que créativité inter-relationnelle existe à tous les niveaux de réalité. Elle se révèle en tant que cosmos dans le déploiement des planètes et des galaxies. Dans l'histoire de la terre, elle se montre dans l'interrelation dynamique de la vie telle qu'elle s'est déployée dans la biosphère. Chaque espèce se ramifie en de nombreuses différences, et sur le plan humain, en de nombreuses races et cultures. Cette diversité n'est pas rupture, mais la base d'une communauté nouvelle et enrichie. Tel est le défi de devenir une communauté globale de vie sur la terre aujourd'hui.

Le processus de créativité est dialectique, un processus de différenciation et de nouvelles occasions de communion. Cette dynamique trinitaire est à la fois créatrice et rédemptrice. Il s'agit du processus qui, à la fois, crée la vie et cherche à corriger les relations déformées afin de rétablir une relation aimante et vivifiante dans de nouveaux contextes. Dieu comme Trinité symbolise tout ce processus : Dieu en tant que matrice créatrice, diversifiante, nourrissante et rédemptrice de la vie cosmique, planétaire, sociale et interpersonnelle.

La création et la présence de Dieu au sein de la création sont un processus dynamique, toujours ouvert à de nouvelles possibilités, à l'espérance qui est en elle. Dans ce potentiel dynamique, les humains ne sont pas séparés du reste de la nature. La vie humaine ne peut exister sans notre intégration à la communauté terrestre entière et au cosmos. Le caractère pécheur de l'humanité engendre des relations déformées l'un avec l'autre, avec les autres humains et tous les êtres terrestres et avec l'univers lui-même. Les relations déformées empoi-

4. Ivone GEBARA, «The Trinity and Human Experience » dans Rosemary RUETHER (éd.), Women Healing Earth : Third World Women on Ecology, Feminism and Religion, Maryknoll, NY, Orbis Press, 1996, p. 13-23. 
sonnent le sol, polluent l'air et les eaux, torturent les corps animaux et humains et nous appauvrissent tous et toutes.

La rédemption est une conversion les uns aux autres et à la terre entière, une conversion qui en même temps nous ouvre à notre plein potentiel, l'épanouissement de la vie dans une relation juste. Nous avons la vie, le mouvement et l'être en Dieu lorsque nous sommes transformés en des moments de relation juste avec les autres humains et avec les autres créatures terrestres, nos semblables. Les animaux et les plantes, les océans et les planètes sont nos ancêtres et nos proches, des manifestations du Saint et les membres d'une seule communauté bien-aimée. Chaque moment est ouvert à Dieu, ouvert à de nouvelles possibilités de devenir ce que nous sommes vraiment et ce que nous sommes appelés à être. Le défi de la théologie éco-féministe est de tisser, à la lumière de l'histoire de la terre aussi bien que des crises engendrées par le péché humain, une vision de la présence divine qui, à la fois, sous-tend et nourrit les processus naturels et nous donne également le pouvoir de lutter contre les excès des puissants et de tendre la main aux victimes afin de créer de nouvelles communautés d'épanouissement mutuel.

\footnotetext{
RÉSUMÉ

Dans cet article, Rosemary Ruether répond au Psalm of Anger to a Patriarchal god en interrogeant le concept de Dieu sousjacent à ce cri. Sa propre vision de Dieu est celle d'une matrice divine sousjacente à toute la réalité et non celle d'un Dieu patriarcal qui justifie la domination des femmes. Cette vision de Dieu comme matrice divine soulève cependant la question de la relation entre la transcendance et l'immanence divines. Ruether rejette la division entre l'immanence et la transcendance qui considère Dieu soit comme un pouvoir distant à l'extérieur et au-dessus de la création, soit comme la fondation immanente de ce qui existe et justifierait du coup les systèmes de domination existants. Ce dont nous avons besoin, c'est d'une synthèse dynamique de l'immanence et de la transcendance qu comprenne Dieu à la fois comme "radicalement autre » que tous les systèmes d'injustice et comme plus près de nous que nous ne le sommes à nous-mêmes. Cette vision sous-tend aussi la théologie écoféministe qui considère tous les êtres comme vivant et liant une relation active Je-Tu les uns avec les autres. Dieu est la matrice dynamique des interrelations entre tous les êtres.
} 


\section{ABSTRACT}

In this article Rosemary Ruether responds to the Psalm of Anger to a Patriarchal god by asking what concept of God underlies this outcry. Her own view of God has been that of a divine matrix underlying all reality, not a distant patriarchal God who sanctions domination of women. But this view of God as divine matrix raises the debate about the relationship between the transcendence and the immanence of God. Ruether rejects the split between immanence and transcendence that sees God either as a distant power outside and over against creation or as the immanent foundation of what exists, in the sense of existing systems of domination. What is needed is a dynamic synthesis of immanence and transcendence that understands God as both "radically other" than all the systems of injustice and also closer to us that we are to ourselves. This view underlies ecofeminist theology also. Ecofeminist theology sees all beings as alive and entering into a lively I-Thou relation with each other. God is the dynamic matrix of interrelationship between all beings. 